\title{
Are We Ready for the Fourth Industrial Revolution?
}

\author{
Hyeoun-Ae Park, President of IMIA
}

According to Professor Klaus Schwab, Founder and Executive Chairman of the World Economic Forum, we are at the beginning of the Fourth Industrial Revolution; this Revolution is fundamentally changing the way we live, work, and relate to one another [1]. In historical context, the First Industrial Revolution -- from the last third of the 18th century -- harnessed water and steam power to mechanize production. The Second Industrial Revolution, a century later, was driven by mass production made possible through electricity. The Third Industrial Revolution began in the 1960s, and used digital technology to automate production.

The Fourth Industrial Revolution, which builds on the Third, is characterized by "a fusion of technologies that are blurring the lines between the physical, digital, and biological spheres" [1]. The Fourth Industrial Revolution is unlike anything we have ever experienced before, in its velocity, scope, and systematic impact. Contrary to the previous Industrial Revolutions, it is progressing at an exponential rather than a linear pace. It is not only changing what and how we do, but also who we are. It involves the transformation of entire systems across countries, companies, industries, and society as a whole. Technologies such as artificial intelligence, the Internet of Things (IoT), cloud computing, social media, data science, 3D printing, connected wearable devices, quantum computing, robotics, and genetics are the driving forces of this Revolution. These transformative technologies will impact all disciplines, economies, businesses, societies, and individuals.

According to the results of a poll by the Economist Intelligence Unit that was released in January 2016, a significant majority of executives surveyed (45\%) believe that healthcare is the sector that will benefit most from the merging of physical, digital, and biological systems [2]. Already, consumer technologies such as smartphones and wearable devices are collecting highly detailed data about health and fitness. Healthcare providers believe that this information has the potential to transform not only individual care, but also medical research [3].

Here are a few examples of how the combined physical, digital, and biological technologies are transforming healthcare. Connected sensors will enable patients to better manage their own health. Novartis developed a digital inhaler that could enable COPD patients to observe data on their own inhalation use in real-time [4]. In the future, patients with respiratory conditions could have sensors embedded into objects in their homes to determine when their breathing becomes labored, and remind them to use an inhaler before hospitalization is necessary. This has the potential to reduce healthcare expenditures incurred due to poor patient compliance with treatments. Artificial intelligence and robotics will present new options for patients and caregivers. For example, researchers at a construction company in Japan are studying how a bendable exoskeleton hugging the patient's waist and thighs with sensors attached to his/her skin can be used to help the patient move [5]. The sensors could detect when the patient's muscles start to move, and direct the machine to support the patient's motion, reducing his/her load's effective weight. This could potentially improve quality of life for the physically disabled. Another example is IBM's Watson project, which is seeking to combine clinical, research, and social data to deliver healthcare solutions in areas ranging from clinical trials to disease management. IBM is working on 
a data streaming app that can be attached to an insulin pump, which could predict - three hours before the event - when a diabetic patient's blood sugar will drop too low [6]. Patients will be able to manage their diabetes more proactively than ever before. Another example is Watson for Oncology [7]. A team of physicians and researchers at Memorial Sloan Kettering trained IBM Watson to develop a cognitive computing system that can analyze large volumes of data including patient health records, medical literature, and clinical trials to offer personalized, evidence-based treatment recommendations for cancer patients. Currently, the system offers recommendations for lung, breast, and colorectal cancers, and it is expanding to gastric-related cancers as well.

Genomics will revolutionize how we diagnose and treat disease. With new technology, it is possible to sequence a person's genome within 24 hours for $\$ 1,000$. This breakthrough will result in an explosion of data that will make preventative and personalized medicine possible. For example, a targeted approach to breast cancer treatment is becoming more of a reality [8]. A patient's disease can be analyzed at a molecular level, allowing the right drug to be delivered to the right target and potentially making the treatment much more effective. As genome editing technology continues to develop, we may be able to precisely delete, repair, or replace the genes that cause currently untreatable diseases, such as cystic fibrosis.Many countries are exploring the potential of telemedicine [9]: that is, monitoring and treating patients remotely via Internet-connected sensors and medical devices. It is hoped that telemedicine will prove especially valuable in the treatment of the elderly with chronic conditions, who will be able to receive healthcare in the comfort of their own homes. It will also be able to bring medical care to communities in remote locations. Looking forward to the future, some of the more sophisticated medical applications emerging from the combination of physical, digital, and biological technologies include drugs with sensors, thought-controlled robotics, and virtual and augmented reality therapy.

There has been great progress in the physical, digital, and biological technologies over the last several years. It's clear that the
Fourth Industrial Revolution could significantly improve the quality of life of billions around the world. However, it will be accompanied by serious challenges for healthcare industries, governments, and individuals [1]: for healthcare industries, adapting to the ongoing digitization of healthcare is already a major challenge. The accelerating velocity, expanded scope, and systematic impact of the Fourth Industrial Revolution will lead to constant surprises and serious disruptions, including - for example - in the area of customer expectations. Digital technologies enable healthcare facilities to better engage with their patients and offer them a superior experience at affordable costs. However, it is challenging to meet the service expectations of digitally empowered patients, and increasingly more difficult to provide such a superior experience to those with sufficient knowledge and alternatives. Product enhancements is another area in which surprises and disruptions will occur. Technological advances are giving rise to a large variety of smart connected products and services, combining sensors, software, data analytics, and connectivity in all kinds of ways. These innovative offerings are restructuring healthcare industry boundaries and are leading to the creation of whole new industries. In the area of collaborative innovations, as a third example, healthcare providers have to become much more innovative in order to better respond to the fast-changing, highly competitive business environment. Collaboration is needed, both within the healthcare sector and beyond - including among customers, partners, startups, universities, and research communities.

Realizing the potential of the Fourth Industrial Revolution, - e.g. IoT, data analytics, and smart devices - requires considerable support and actions from policy makers [1]. These include policies to protect the privacy and rights of consumers and healthcare businesses, stronger security requirements for critical devices and systems, incentives that promote fair data sharing across healthcare providers, and new regulations to help us deal with increasingly intelligent machines.

Support from policy makers is also required to help address job creation, one of the toughest challenges facing nations around the world. Though revolution means a risk of job displacement in every industry, at the same time there will be a growing demand for certain skilled workers. As we enter the Fourth Industrial Revolution, those with skills and expertise in artificial intelligence and machine-learning, robotics, nanotechnology, 3-D printing, genetics and biotechnology, and big data analytics are highly sought after [10]. There is no doubt that decades from now, the skills and expertise that will be the most in demand will yet again be different. This means we need to keep educating ourselves as the world around us inevitably progresses.

However, in addition to creating economic opportunities, the Fourth Industrial Revolution exacerbates certain pre-existing inequalities, such as the digital divide. Robotics and machine intelligence may replace many of today's jobs. Those who are digitally marginalized - such as the elderly and the poor - could experience the digital divide more severely than ever before. It is the responsibility of policy makers to reduce the digital divide.

The Fourth Industrial Revolution will change not only what and how we do but also who we are. It will affect our identity and all associated issues: how we conceptualize privacy and ownership, how we consume, how we allocate time for work and leisure, how we develop skills, plan careers, and form relationships with people [1]. On the one hand, the Revolution allows us to conceptualize a quantified and augmented self, based on the data that our technologies collect about ourselves. On the other hand, it is crucial to maintain human touch and social interaction, even in a world where "information is power" and people increasingly connect online.

Schwab writes in his concluding paragraph, "in the end, it all comes down to people and values. [...] We need to shape a future that works for all of us by putting people first and empowering them. In its most pessimistic, dehumanized form, the Fourth Industrial Revolution may indeed have the potential to robotize humanity and thus to deprive us of our heart and soul. But as a complement to the best parts of human nature - creativity, empathy, stewardship - it can also lift humanity into a new collective and moral consciousness based on a shared sense of destiny. It is incumbent on us all to make sure the latter prevails [1]." 
The end results of the Fourth Industrial Revolution will be hugely beneficial for all of us, as we'll be connecting patients to each other, to their caregivers, and clinicians with combined physical, digital, biological technologies. We will be able to understand how the human genome, phenome, and exposome impact our health in more detail, and be able to provide tailored care in an affordable way. It will mean offering better healthcare, improving health, and extending the life-spans of billions of people around the world, many of whom currently have little to no access to healthcare.

The fusion of physical, digital, and biological technologies is not only a product of science and engineering, but also a product of values and institutions. IMIA as an international organization on health and biomedical informatics needs a shared vision of how it can better benefit economies, societies, and individuals. Collaboration with industry, government, and academia is essential to create a shared vision of the future. The speed and scope of the Fourth Industrial Revolution forces us to rethink how IMIA as an organization creates value.

IMIA has much to gain from the coming revolution. As such it must play a pivotal role in ensuring the wave of change benefits the whole of humanity. There are various ways for IMIA to become involved in the Fourth Industrial Revolution.

One avenue will be Medinfo, IMIA's world congress on health and biomedical informatics. Medinfo provides opportunities to share and exchange ideas and research results, as well as to hold formal meetings and facilitate the informal networking of members. I look forward to the IMIA community discussing this vision for and tackling some of the opportunities and challenges of - the Fourth Industrial Revolution at the coming Medinfo conference.
Medinfo 2017, with the theme "precision healthcare through informatics", will be a perfect opportunity for us to engage with such questions; it will be held from August 21 to 25 in Xiamen, China.

Another avenue would be a Working Group (WG) or Special Interest Group (SIG). IMIA pursues its scientific activity in specific fields of the wider domain of health and biomedical informatics through WGs and SIGs. Activities of WGs and SIGs include organizing business meetings at IMIA conferences or IMIA regional meetings, publishing papers related to WG activity written by members of WGs and SIGs, and collaborating with other organizations or IMIA regional or member societies within IMIA. IMIA can form a WG or SIG to address a particular set of issues related to the Fourth Industrial Revolution in healthcare.

IMIA publications can be used as platforms for sharing and exchanging topics on the Fourth Industrial Revolution in healthcare, such as the use of transformative technologies, impact of the technologies on health, and other related issues. IMIA publishes the annual IMIA Yearbook of Medical Informatics and three official journals: the International Journal of Medical Informatics, Methods of Information in Medicine, and Applied Clinical Informatics.

We can also ensure IMIA's involvement in the Fourth Industrial Revolution by creating a shared vision of the future, identifying its importance as a movement and declaring our commitment to it in IMIA's strategic plan. IMIA also needs to revise the IMIA Code of Ethics of Health Information Professionals to ensure that it covers the ethical issues related to the Fourth Industrial Revolution

I hope IMIA can play an important role in helping to ensure that the Fourth Industrial Revolution benefits populations we care about.

\section{References}

1. Klaus Schwab. The Fourth Industrial Revolution. World Economic Forum; 2015.

2. The Economist Intelligence Unit Flash poll, Healthcare to benefit most from the Fourth Industrial Revolution, Executives predict. The Economist Intelligent Unit Limited. January 2016, Available at: https://www.eiuperspectives. economist.com/technology-innovation/healthcare-benefit-most-fourth-industrial-revolution-executives-predict

3. The Economist Intelligence Unit. Power to the patients: How mobile technology is transforming healthcare. The Economist Intelligent Unit Limited; 2015. Available at: https://www. eiuperspectives.economist.com/sites/default/files/ HowMobileisTransformingHealthcare.pdf

4. Wedzicha JA, Banerji D, Chapman KR, Vestbo J, Roche N, Ayers T, et al. Indacaterol-Glycopyrronium versus Salmeterol-Fluticasone for COPD. N Engl J Med 2016;374:2222-34.

5. Schlesinger JM, Martin A. Graying Japan tries to embrace the golden years - Entrepreneurs are exploring robotics and other innovations to unleash the potential of the elderly. The 2050 Demographic destiny: Aging gracefully. The Wall Street Journal, November 29, 2015. Available at: http://www.wsj. com/articles/graying-japan-tries-to-embrace-thegolden-years-1448808028

6. Forbes. IBM shows off 'empathic' robot, offers Watson to diabetics; 2016.

7. Medical Marketing \& Media. How Watson for Oncology is advancing cancer care. April 19, 2016. Available at: http://www.mmm-online.com/ technology/how-watson-for-oncology-is-advancing-cancer-care/article/490336/

8. Cho SH, Jeon J, Kim SI. Personalized medicine in breast cancer: A systematic review. J Breast Cancer 2012;15(3):265-72.

9. Oh J-Y, Park Y-T, Jo EC, Kim S-M. Current status and progress of telemedicine in Korea and other countries. Healthc Inform Res 2015;21(4):239-43.

10. World Economic Forum. The future of jobs: Employment, skills and workforce strategy for the Fourth Industrial Revolution. January 2016.

http://dx.doi.org/10.15265//Y-2016-052 